\title{
Neue Hoffnung für Reizdarmpatienten
}

\author{
Ein neu entwickelter Opioidrezeptormodulator bewirkte in einer Phase-III-Studie über sechs \\ Monate hinweg einen signifikanten Rückgang der Durchfallepisoden bei Reizdarmsyndrom.
}

\begin{abstract}
— Das Reizdarmsyndrom ist in allgemeinärztlichen Praxen ein häufiger Beratungsanlass. Die Behandlungsoptionen - insbesondere bei Reizdarmsyndrom mit vorwiegender Diarrhö - beschränken sich im Wesentlichen auf diätetische Maßnahmen. Bei der Erforschung neuer Therapieoptionen stehen seit Langem die $5-\mathrm{HT}_{3}$ - und die Opioidrezeptoren im Zentrum des Interesses. Jüngstes Kind der Familie der Opioidrezeptormodulatoren ist Eluxadolin, ein gemischter $\mu$ - und $\kappa$-Opioidrezeptoragonist und $\delta$-Opioidantagonist.

Für eine herstellerfinanzierte PhaseIII-Studie wurden über 2.400 Erwachsene mit diarrhöbetontem Reizdarmsyndrom über 26 Wochen hinweg mit 75 oder 100 mg Eluxadolin oder Placebo

Rückgang der Bauchschmerzen und eine Verbesserung der Stuhlkonsistenz am selben Tag an mindestens der Hälfte der Tage erlebte.

In den ersten zwölf Wochen erreichten $23,9 \%$ der Patienten unter der 75-mgDosis und 25,1\% unter der 100-mg-Dosis Eluxadolin den primären Endpunkt. Mit Placebo waren es lediglich $17,1 \%$. In einem zweiten Schritt wurden die Wochen 1 bis 26 betrachtet. Über diese Zeit betrugen die Werte $23,4 \%$ für die 75 -mgDosis, 29,3\% für die 100-mg-Dosis und 19,0\% für Placebo. Die häufigsten Nebenwirkungen bei beiden Dosen waren Übelkeit, Bauchschmerzen und Obstipation, die unter Verum in einer Häufigkeit zwischen 5 und 8\%, unter Placebo zwischen 2 und $4 \%$ auftraten.
\end{abstract} behandelt. Primärer Studienendpunkt war der Anteil von Patienten, die einen
- Lembo AJ et al. Eluxadoline for irritable bowel syndrome with diarrhea. N Engl J Med. 2016;374:242-53

\section{KOMMENTAR}

Welch starken Einfluss psychische Faktoren beim Reizdarmsyndrom haben, wird aus dem relativ kleinen Unterschied zwischen den Ergebnissen unter der Substanz und unter Placebo deutlich. Da es sich um eine Störung handelt, die zwar die Lebensqualität stark beeinträchtigen kann, aber prognostisch günstig verläuft, sind unerwünschte Arzneimittelwirkungen von großer Wichtigkeit. Wir haben in den letzten Jahren viele Substanzen kommen und gehen sehen, die anfangs hoch gelobt wurden, nach kurzer Zeit aber wegen gastrointestinaler und kardialer Nebenwirkungen wieder verschwunden sind. Eluxadolin hat vor diesem Hintergrund den Vorteil, dass es fast ausschließlich lokal an der Darmwand wirksam ist. Daher sind systemische Nebenwirkungen kaum zu erwarten.

Prof. Dr. med. H. S. FüeßI

\section{Am ganzen Körper entstellt - durch Jod}

Ein 57-jähriger Mann kam wegen einer seit 12 Stunden bestehenden Hämaturie in die Nothilfe. Kurz vorher hatte er ein intravenöses Pyelogramm bekommen. Wenige Stunden danach traten im Gesicht und an den Ohren flächenhaft konfluierende Bläschen und Pusteln auf (Abb. A, B). Ähnliche Veränderungen entstanden auch am Stamm, an Armen und Beinen. Im Blutbild fand sich eine leichte Eosinophilie von $8 \%$ bei normaler Gesamtleukozytenzahl. Die Histologie der Haut ergab eine pseudoepitheliomatöse Hyperplasie, eine epidermale Nekrose und diffuse leukozytäre Infiltrate der Dermis. Die mikrobiologischen Befunde waren negativ. Anhand der Vorgeschichte und des klinischen Bildes stellte man die Diagnose eines Jododerms.
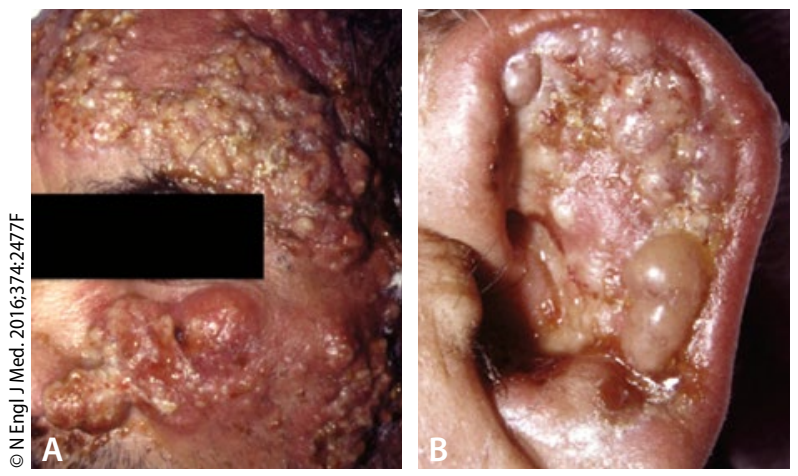

Flächenhaft konfluierende Bläschen und Pusteln im Gesicht $(A)$ und an den Ohren (B).

Dabei handelt es sich um eine Hypersensitivitätsreaktion gegen Jod, das im verabreichten Kontrastmittel reichlich vorhanden war.

Die Pathogenese dieser Veränderung ist nicht ganz klar. Man nimmt eine verminderte Clearance für Jod und die Auslösung einer neutrophilen Degranulation an. Häufigste Ursache ist die Gabe von jodhaltigem Kontrastmittel, es gibt aber auch Berichte über die Auslösung durch oral zugeführtes oder topisch appliziertes Jod. Unter einer Therapie mit Thalidomid bildeten sich die Hautläsionen innerhalb von vier Wochen vollständig zurück. Die Ursache der nach wenigen Tagen spontan sistierten Hämaturie konnte nicht gefunden werden. Die Nierenfunktion des Patienten war nicht beeinträchtigt.

Prof. Dr. med. H. S. FüeßI

- Chalela JG, Aguilar L (juanguibucaro@gmail.com). Iododerma from contrast material. N Engl J Med. 2016;374:2477F 